\title{
Treatment of Advanced Malignant Uterine Perivascular Epithelioid Cell Tumor with mTOR Inhibitors: Single-institution Experience and Review of the Literature
}

\author{
KRISTEN D. STARBUCK ${ }^{1,2^{*}}$, RICHARD D. DRAKE ${ }^{3 * *}$, G. THOMAS BUDD ${ }^{4}$ and PETER G. ROSE ${ }^{3}$ \\ ${ }^{1}$ Department of Obstetrics and Gynecology, ${ }^{3}$ Division of Gynecologic Oncology, and \\ ${ }^{4}$ Department of Hematology and Oncology, Cleveland Clinic, Cleveland, OH, U.S.A.; \\ ${ }^{2}$ Department of Obstetrics and Gynecology, Metrohealth Medical Center, Cleveland OH, U.S.A.
}

\begin{abstract}
Uterine perivascular epithelioid cell tumors (PEComas) are rare mesenchymal tumors. Many have malignant behavior, and no successful treatment strategy has been established. Identification of mutations in the tuberous sclerosis 1 (TSC1) and TSC2 genes producing constitutive activation of the mammalian target of rapamycin (mTOR) pathway presents an opportunity for targeted therapy. Patients with advanced malignant uterine PEComa treated with mTOR inhibitors were identified and records were retrospectively reviewed for treatment response based on radiographic assessment. Three patients with advanced uterine PEComas underwent debulking surgery followed by mTOR inhibitor therapy; two had a complete response to therapy and disease in one patient progressed. Conclusion: Given the absence of effective therapies for malignant uterine PEComas, targeting the MTOR pathway is a logical strategy to pursue given the known pathobiology involving the Tuberous Sclerosis complex. Treatment of malignant uterine PEComas with mTOR inhibitors was effective in two out of three patients after surgical resection, with durable response.
\end{abstract}

Perivascular epithelioid cell tumors (PEComas) are a relatively recently described medical entity, first recognized

This article is freely accessible online.

*Currently at: Roswell Park Cancer Institute, Division of Gynecologic Oncology, Buffalo, NY, U.S.A.; **Currently at: Texas Oncology, Houston TX, U.S.A.

Correspondence to: Kristen D Starbuck, MD, Roswell Park Cancer Institute, Division of Gynecologic Oncology, Elm and Carlton Streets, Buffalo NY 14263, U.S.A. Tel: +1 7168453910, e-mail: Kstarbuck79@gmail.com

Key Words: PEComa, mTOR inhibitor, perivascular epithelioid cell tumor, sirolimus, temsirolimus. as a distinct group of tumors with specific morphologic and immunohistochemical characteristics by the World Health Organization in 2002 (1). They have myomelanocytic differentiation, epithelioid morphology, and a propensity for perivascular distribution. PEComas demonstrate smooth muscle markers (smooth muscle actin), melanocytic markers (human melanoma black 45 (HMB45), melan-A), and cathepsin K (2). These tumors lack expression of protein S100 , cytokeratin and desmin, which differentiates them from melanomas, clear-cell sarcomas and smooth-muscle tumors (1-6). PEComas are mesenchymal neoplasms belonging to a group of rare tumors including angiomyolipoma (AML), clear-cell sugar tumor, and lymphangioleiomyomatosis (LAM). The uterus is the most commonly reported site of PEComa-Not Otherwise Specified, comprising 38/100 reported cases in this group (4).

The clinical behavior of uterine PEComas in the literature is varied and ranges from benign, through uncertain malignant potential, to aggressive malignant behavior with a propensity for metastasis. Pathological features associated with aggressive, malignant behavior include tumor size $>5$ $\mathrm{cm}$, coagulative necrosis, high mitotic activity (>1/10 highpower fields), cytologic atypia, lymphovascular invasion, and infiltrativeness (7). Tumors in the PEComa group are rare and usually sporadic. However, they can be associated with tuberous-sclerosis complex (TSC), and both sporadic and TSC-related cases demonstrate similar genetic alterations of the TSC1 or TSC2 locus $(8,9)$. The TSC1 and TSC2 gene products form a complex which negatively regulates mammalian target of rapamycin complex 1 (mTORC1) and therefore PEComas as well as LAM and AML, have constitutive activation of the mTOR pathway. Given the poor prognosis of malignant PEComas and the lack of effective therapies, there is increasing research into targeting molecular pathways as treatment alternatives. Recently, a number of reports have described the use of sirolimus, a drug targeting the mTOR pathway, to treat several tumor types 
including AML, LAM and PEComas (9-11). The response in these patients is encouraging and warrants further investigation into the use of mTOR inhibitors in TSCassociated tumors. Here we report our institution's experience with advanced uterine PEComas treated with mTOR inhibitors.

Patients diagnosed with advanced malignant uterine PEComas treated with mTOR inhibitors were retrospectively identified and records reviewed on an active protocol (IRB 7419). Three patients with advanced uterine PEComas underwent debulking surgery followed by mTOR inhibitor therapy and met the inclusion criteria (pathological diagnosis of malignant uterine PEComa; advanced disease at time of presentation; treatment with an mTOR inhibitor); two had a complete response to therapy and disease in one patient progressed. These cases are described below.

\section{Case Reports}

Patient 1 is a 30 -year-old individual with a past medical history of tuberous sclerosis who presented to a Cleveland Clinic satellite hospital in October 2012 with worsening abdominal pain. She was found to have an acute abdomen with profound anemia (hemoglobin $4.4 \mathrm{mg} / \mathrm{dl}$ ) and a $26.85 \times 15.9 \times 29.2 \mathrm{~cm}$ complex pelvic mass on computed tomographic (CT) scan. The patient was given fluid resuscitation, transfused multiple units of packed red blood cells and underwent emergency exploratory laparotomy, total abdominal hysterectomy, L salpingo-oophorectomy, omentectomy, and mesenteric resection by a staff Gynecologic Oncologist. Findings at the time of surgery included a large mass originating from the left side of the uterus and a $4000 \mathrm{cc}$ hemoperitoneum. Pathology revealed the mass to be consistent with a malignant PEComa. Postoperatively she was transferred to the Intensive Care Unit for stabilization and was discharged on postoperative day 5 .

The patient was started on gemcitabine $\left(900 \mathrm{mg} / \mathrm{m}^{2}\right.$ day 1 and 8 every 3 weeks) and docetaxel $\left(100 \mathrm{mg} / \mathrm{m}^{2}\right.$ on day 8 every 3 weeks) for three cycles, however, her disease progressed with suspected bone metastases and therapy was changed to temsirolimus $25 \mathrm{mg}$ weekly. She responded well initially; however, after five cycles she developed a large pelvic hematoma. Imaging revealed a pelvic mass separate from the hematoma, therefore Medical Oncology recommended changing therapy to the closely related mTOR inhibitor sirolimus. She was treated with a $6 \mathrm{mg}$ loading dose followed by $3 \mathrm{mg}$ daily. The pelvic mass decreased on imaging and as of October 2015, there had been no signs of progression or metastatic disease, 36 months from her original presentation.

Patient 2 is a 43-year-old individual who presented to a hospital in Saudi Arabia in June 2012 with severe abdominal pain and syncope. She had an acute abdomen so an emergency exploratory laparotomy was performed with resection of extensive tumor. The patient required urgent blood transfusion for significant hemoperitoneum, requiring 8 units packed red blood cells and 8 units fresh frozen plasma. She remained in the Intensive Care Unit for 3 days with progressive improvement and was discharged in a stable condition. Histopathology showed a high-grade endometrial stromal sarcoma. Chemotherapy was initiated with carboplatin and paclitaxel for three cycles in Saudi Arabia, and subsequently the patient sought a second opinion at our Institution which led to transfer of care after the third cycle. Imaging in January 2013 demonstrated a mass superior and left of the vaginal cuff extending into the left pelvis. This mass appeared unchanged compared to the prior imaging the patient had brought with her and was thought to represent postoperative changes. Chest imaging revealed no evidence of metastasis. The patient was continued on her fourth of an intended six cycles of carboplatin and paclitaxel. Pathology review at our institution demonstrated an extensively infiltrating malignant PEComa of the endometrium and myometrium involving the omentum, transverse colon, and segmental excisions. Her therapy was therefore switched to temsirolimus at $25 \mathrm{mg}$ weekly. In June 2013, after 4 months of treatment, CT imaging of the chest, abdomen, and pelvis revealed no evidence of disease. There was no sign of recurrence at 12 months from completion of treatment, and the patient subsequently returned to Saudi Arabia.

Patient 3 is a 64-year-old female who underwent a laparoscopic hysterectomy and bilateral salpingooophorectomy with morcellation in October 2013. Pathology demonstrated a uterine leiomyosarcoma, and she was treated with three cycles of gemcitabine $\left(900 \mathrm{mg} / \mathrm{m}^{2}\right.$ day 1 and 8 every 3 weeks) and docetaxel $\left(100 \mathrm{mg} / \mathrm{m}^{2}\right.$ day 8 every 3 weeks). At that time positron-emission tomography

CT was negative and therapy was discontinued. The patient experienced recurrence in August 2014 with multiple abdominal nodules. Pathology review of the original specimen at our hospital demonstrated architecture and immunophenotype consistent with a malignant uterine PEComa. In September 2014, she underwent exploratory laparotomy with tumor cytoreduction to microscopic residual disease, including ileal and rectosigmoid resections. Pathology was consistent with recurrent malignant PEComa. The patient was started on $25 \mathrm{mg}$ of temsirolimus weekly from 14th October 2014 until 13th January 2015, and imaging after 3 months of treatment revealed interval progression of disease with enlarging pelvic masses, an enlarged para-aortic lymph node, and several indeterminant lung nodules suspicious for metastases. Temsirolimus was therefore discontinued. In February 2015, the patient developed a malignant bowel obstruction that failed conservative management, and she subsequently died in March 2015. 


\section{Discussion}

Most patients with aggressive PEComas and extensive dissemination have a uniformly poor prognosis. Current chemotherapy strategies have not been successful in advanced disease, and recent focus has been drawn to targeting the mTOR pathway, which is up-regulated in PEComas. There are several reports in the medical literature describing a total of seven patients with uterine or 'gynecologic' PEComas who received treatment with mTOR inhibitors, with varying degrees of success [50\%-100\% response rates (8-12)]. Given this robust response and the plausible theoretical basis behind it, we proceeded to manage three cases of advanced PEComas with mTOR inhibitors. Two out of three patients had a significant and prolonged response to treatment with mTOR inhibitors. All patients had advanced disease at the time of presentation to our service, two of which led to acute, life-threatening illness. All three patients underwent extensive debulking surgery and all were treated with mTOR inhibitors; this treatment strategy was both well tolerated and to date has been successful in two out of the three patients with no sign of recurrence or progression. Patient 3, whose disease progressed, had a tumor lacking smooth muscle actin or desmin positivity, which has been noted in previous studies in PEComas with a transcription factor binding to IGHM enhancer 3 (TFE3) fusion gene product instead of mTOR pathway alterations (13), and this may explain the lack of response to temsirolimus. Additionally, approximately $75 \%$ of sporadic PEComas have alterations in TORC2, which in renal cell carcinomas is relatively more resistant to mTOR inhibitors $(14,15)$. Testing for the TFE3 fusion gene product (Xp11) should be considered in patients with malignant uterine PEComas, as combination treatment with vascular endomethial growth factor- or phosphatidylinositol-3-kinase-directed biological therapies may be more effective in this group.

Notably, patient 1 was treated with temsirolimus and responded, and when her disease progressed, therapy was switched to sirolimus, with a complete response and the patient remains disease-free. As far as we are aware, this is the first report of a patient with progression of PEComa on therapy with mTOR inhibitor which was successfully rescued with another mTOR inhibitor. To date in literature, treatments of cases with mTOR inhibitors which subsequently progressed were changed to another class of chemotherapy or treatment was stopped altogether Although it is possible that PEComas develop dependence on alternative pathways for tumorigenesis, the fact that they often demonstrate mutations in the mTOR pathway make it worth considering a trial of an alternative mTOR inhibitor. Currently, post-operative treatment of PEComas with mTOR inhibitors is still preliminary and is limited to a few reports. We suggest that prospective cooperative trials, as well as further molecular profiling, including of TFE3 fusion and mTOR pathway genes, are needed to more fully investigate the clinical benefit of this strategy in patients with malignant uterine PEComas, which has the potential to significantly impact the prognosis of such patients.

\section{Funding}

No financial disclosures or funding support for this study.

\section{References}

1 Folpe AL: Neoplasms with perivascular epithelioid differentiation (PEComas). In: World Health Organization Classification of Tumors: Pathology and Genetics of Tumors of Soft Tissue and Bone (Fletcher CDM, Unni KK, Mertens F (eds.)) Lyon: IARC Press pp. 221-222, 2002.

2 Martignoni G, Pea M, Zampini C, Brunelli M, Segala D, Zamboni G and Bonetti F: PEComas of the kidney and of the genitourinary tract. Semin Diagn Pathol 32: 140-159, 2015.

3 Folpe AL, Goodman ZD, Ishak KG, Paulino AFG, Taboada EM, Meehan SA and Weiss SW: Clear cell myomelanocytic tumor of the falciform ligament/ligamentum teres. Am J Surg Pathol 24: 1239-1246, 2000.

4 Armah HB and Parwani AV: Malignant perivascular epithelioid cell tumor of the uterus with late renal and pulmonary metastases: a case report with review of the literature. Diagn Pathol 2: 45, 2007.

5 Vang $\mathrm{R}$ and Kempson RL: Perivascular Epithelioid Mesenchymal Tumor ('PEComa') of the Uterus: a subset of HMB-45-positive epithelioid mesenchymal neoplasms with an uncertain relationship to pure smooth muscle tumors. Am J Surg Pathol 26: 1-13, 2002.

6 Bonetti F, Martignoni G, Colato C, Manfrin E, Gambacorta M, Faleri M, Bacchi C, Sin Vai-Chong, Wong Nim-Lai, Coady M and Kwok-cheung JC: Abdominopelvic sarcoma of perivascular epithelioid cells. Report of four cases in young women, one with tuberous sclerosis. Mod Pathol 14: 563-568, 2001.

7 Fadare O: Perivascular Epithelioid Cell Tumor (PEComa) of the Uterus: An outcome-based clinicopathologic analysis of 41 reported cases. Adv Anat Pathol 15: 63-75, 2008.

8 Dickson MA, Schwartz GK, Antonescu CR, Kwiatkowski DJ and Malinowska IA: Extrarenal perivascular epithelioid cell tumors (PEComas) respond to mTOR inhibition: clinical and molecular correlates. Int J Cancer 132: 1711-1717, 2013.

9 Wagner AJ, Malinowska-Kolodzie I, Morgan JA Qin W, Fletcher $\mathrm{CD}$, Vena N Ligon AH, Antonescu CR, Ramaiya NH, Demetri GD, Kwiatkowski DJ and Maki RG: Clinical activity of mTOR inhibition with Sirolimus in malignant perivascular epithelioid cell tumors: targeting the pathogenic activation of mTORC1 in tumors. J Clin Oncol 28: 835-840, 2010.

10 Italiano A, Delcambre C, Hostein I, Cazeau AL, Marty M, Avril A Coindre JM and Bui B: Treatment with the mTOR inhibitor Temsirolimus in patients with malignant PEComa. Ann Onc 21: 1135-1137, 2010.

11 Benson C, Vitfell-Rasmussen J, Maruzzo M, Fisher C, Tunariu N, Mitchell S Al-Muderis O, Thway K, Larkin J and Judson I: A retrospective study of patients with malignant PEComa receiving treatment with Sirolimus or Temsirolimus: The Royal Marsden Hospital experience. Anticancer Res 34: 3663-3668, 2014. 
12 Ghosh I, Arun I, Sen S and Mishra L: Metastatic perivascular epithelioid cell tumor responding to mammalian target of rapamycin inhibition. Indian J Med Paediatr Oncol 35: 99-102, 2014.

13 Argani P, Aulmann S, Illei PB Netto GJ, Ro J and Cho HY: A distinctive subset of PEComas harbors TFE3 gene fusions. Am J Surg Pathol 34: 1395-1406, 2010.

14 Thway K and Fisher C: PEComa: morphology and genetics of a complex tumor family. Ann Diagn Pathol 19: 359-368, 2015.
15 Husseinzadeh $\mathrm{N}$ and Husseinzadeh HD: mTOR inhibitors and their clinical application in cervical, endometrial and ovarian cancers: A critical review. Gynecol Oncol 133: 375-381, 2014.

Received July 18, 2016

Revised August 14, 2016

Accepted August 18, 2016 